\title{
Rapid Coagulation of Polystyrene Latex in a Stopped-Flow Spectrophotometer
}

\author{
J. W. TH. LICHTENBELT, ${ }^{\mathfrak{1}}$ C. PATHMAMANOHARAN, AND P. H. WIERSEMA \\ Van't Hoff Laboratorium, Transitorium 3 van de Rijksuniversiteit, Utrecht, The Netherlands
}

Received February 1, 1974; accepted April 18, 1974

\begin{abstract}
With a stopped-flow method the rapid coagulation by electrolyte of several polystyrene latices is measured. By extrapolating back to zero time the initial process of two single particles forming a doublet is observed. We find an average rate constant $k_{11}=6.0 \times 10^{-12} \mathrm{p}^{-1} \mathrm{~cm}^{3} \mathrm{sec}^{-1}$ at $20^{\circ} \mathrm{C}$, where $\mathrm{p}$ is the number of particles; this constant is lowered to $4.9 \times 10^{-12} \mathrm{p}^{-1} \mathrm{~cm}^{3}$ $\sec ^{-1}$ when we assume that the particle diameters are $5 \%$ lower than the Dow values. These values are equal to 56 and $46 \%$, respectively, of the von Smoluchowski values. Using the theory of hydrodynamic interaction, this gives Hamaker constants $A=7.0 \times 10^{-21} \mathrm{~J}$ or $1.5 \times 10^{-21} \mathrm{~J}$, respectively, in good agreement with theoretical predictions.
\end{abstract}

\section{INTRODUCTION}

The rate of rapid coagulation of lyophobic colloids has been measured in the past fifty years with various methods, such as direct counting in the ultramicroscope $(1 \mathrm{a}, 2,3)$ or in the Coulter Counter $(4,5)$, light scattering $(6,7)$, and turbidimetry $(2,8)$. During coagulation many processes occur; they can be described in general as the combination of an aggregate of $m$ monomers with an aggregate of $n$ monomers to form one of $m+n$ monomers. Since most methods of observation give insufficient information to follow each reaction separately, and since the theory leads to the expectation that rate constants are only weakly dependent on $m$ and $n$, all rate constants are usually assumed to be equal. However, in the very beginning there is only one process: two single particles form one double particle. Detailed theoretical predictions are available only for the rate constant of this process. The theory considers brownian motion (9), interparticle forces $(1 \mathrm{~b}, 10)$, and hydrodynamic interaction $(11,12)$.

1 Present address: AKZO Research Laboratories, Arnhem, The Netherlands.
We observed this initial stage of coagulation immediately after mixing equal volumes of monodisperse polystyrene latex and electrolyte in a stopped-flow spectrophotometer. Especially, rapid coagulation was observed for a range of particle diameters.

\section{MATERIALS AND METHODS}

We used a Durrum-Gibson stopped-flow spectrophotometer. This instrument has been designed to measure changes of consumptive absorption with time. The angle of illumination of the cuvette and the angle of acceptance of the photomultiplier tube are rather large $\left(3^{\circ}\right)$ for the measurement of turbidity caused by light scattering, when we want to prevent primary and multiply scattered light from reaching the photomultiplier tube. Thus these angles were diminished to less than $1^{\circ}$ by introducing diaphragms into the light path before and behind the cuvette in a similar way as described by Heller and Tabibian (13) for a Beckman DU spectrophotometer. As a light source we used a tungsten lamp fed by a stabilized dc power supply, with interchangeable Balzer interference filters as monochroma- 
tors (wavelengths $438,546,673$, and $752 \mathrm{~nm}$; bandwidth $12 \mathrm{~nm}$ ). The output (anode) current from the photomultiplier tube was passed to ground through a $100-k \Omega$ resistor, and the resulting voltage was fed to a PAR Model 113 low-noise preamplifier, and from there to a storage oscilloscope. The signal is proportional to the light transmission $T$; it appeared on the oscilloscope screen as a function of time and was photographed with a Polaroid camera. We used cuvettes with a 20- or 2.4-mm light path, made of blackened material (polychlorotrifluoroethylene) in order to minimize reflections at the cell walls.

The mixing dead time is a few milliseconds. Reactions can be followed immediately thereafter, and on the same time scale, if they cause large changes of transmission. This usually occurs with chemical reactions that have colored reactants or products. In our coagulation experiments a much longer time of observation was necessary in order to get a change of transmission (about 1\%) large enough to be measured with some accuracy in spite of the noise of the signal. This experimental restriction set a lower limit to our time of observation.

An upper limit was dictated by our own requirement to measure before one-tenth of the reaction half-time $\left(T_{\frac{1}{2}}\right)$ would be over, as later the formation of triplets and larger particles becomes appreciable. $T_{\frac{1}{2}}$ is the time that is necessary for the total number of particles to diminish by a factor of 2 ; it is inversely proportional to the concentration according to the relation $(1 \mathrm{c})$

$$
T_{\frac{1}{2}}=2 / k_{11} N_{1}
$$

where $k_{11}$ is the reaction rate constant of two primary particles forming a doublet and $N_{1}$ is the initial concentration of primary particles. We performed all measurements at $t=0.07 T_{\frac{1}{2}}$ and extrapolated back to $t=0$. Our times of measurement ranged from $0.04 \mathrm{sec}$ with the smallest particles to $3 \mathrm{sec}$ with some of the larger particles, while $T_{\frac{1}{2}}$ ranged from about 0.6 to $50 \mathrm{sec}$.

Theoretical calculations (15) show that at $t=0.07 T_{\frac{1}{2}}$ coagulation is at its steady state; it has passed the non-steady-state in which coagulation is even faster than in the steady state.

The instrument has a single-beam optical system and has a poor long-term stability. Thus very slow processes (which occur in dilute sols) could not be followed. In practice a minimum change in transmission of about $0.2 \% \mathrm{~T} / \mathrm{sec}$ was required. In addition, trans-' mission below $30 \% T$ could not be measured accurately, so that an upper limit was set to the sol concentration, especially for large particles. These two requirements set an upper limit of about $350 \mathrm{~nm}$ to the diameter of the latices we could use.

Air bubbles in the cuvette were a chronic problem. They made the signal unstable during the first few seconds after mixing. Usually we managed to get rid of the bubbles by flushing the system with air-free water. As a control experiment we mixed latex with water in the cuvette and watched the transmission on the same time scale as the experiment with electrolyte.

Monodisperse Dow polystyrene latices with diameters of $91 \mathrm{~nm}$ (Serva 41907), $109 \mathrm{~nm}$ (Dow LS-1044-E), 176 nm (Dow LS-1045-E), $234 \mathrm{~nm}$ (Dow LS-1047-E), $312 \mathrm{~nm}$ (Dow LS1018-E, Serva 41927), and $357 \mathrm{~nm}$ (Dow LS-1010-E) were used as supplied. From one quantity of latex (diameter $234 \mathrm{~nm}$ ) the emulsifier (sodium dihexyl sulfosuccinate) was removed by ion exchange (14).

The absence of aggregates was ensured with the following methods. (a) Centrifugation with a swinging bucket rotor in a preparative ultracentrifuge: A thin layer of latex is brought upon an ethanol-glycerol mixture with a density gradient of $1.00-1.05 \mathrm{~g} \mathrm{ml}^{-1}$. After centrifugation, doublets would be visible as a separate layer because they sediment faster than singlets do. The density gradient prevents convection during centrifugation; the viscosity of the mixture reduces convection during handling afterwards. (b) Centrifugation with a zonal rotor in a preparative ultracentrifuge in a density gradient of water-glycerol 
mixtures of $1.01-1.04 \mathrm{~g} \mathrm{ml}^{-1}$. (This is also a very good preparative method for monodisperse sols; the product contains glycerol, which can be removed by dialysis.)

As a preliminary experiment we coagulated latex with varying electrolyte concentrations. The main experiments were done with electrolyte concentrations well beyond the critical coagulation concentration, where the plateau of rapid coagulation is reached. The electrolyte concentrations-after mixing with latex-were $\mathrm{LaCl}_{3} 0.0058$ mole $\mathrm{dm}^{-3}, \mathrm{BaCl}_{2}$ and $\mathrm{MgCl}_{2}$ 0.05 mole $\mathrm{dm}^{-3}$, and $\mathrm{NaCl} 0.4$ mole $\mathrm{dm}^{-3}$. Most experiments were done with $\mathrm{BaCl}_{2}$, as other salts gave the same results. With $\mathrm{NaCl}$, observation was possible only after $5 \mathrm{sec}$, as incomplete mixing gave a higher initial turbidity. This was caused by the high concentration and, consequently, the high density of this salt solution necessary for rapid coagulation.

Latex from which all emulsifier had been removed by ion exchanging gave the same results as latex with emulsifier. As the ion exchange treatment is a rather tiresome procedure and moreover, as it destabilizes latices with low surface charges, we used latex with emulsifier in all further experiments.

When one cuvette was used, the latex concentration could be varied within narrow limits only, because the transmission should not be too low or too high-between 30 and $70 \%$. The concentration could be varied one order of magnitude by using two different cuvette lengths: 2.4 and $20 \mathrm{~mm}$. Within this narrow range we did not find variation of the rate constant with the sol concentration.

Rate constants were calculated with Eq. [6] of Ref. (16):

$$
k_{11}=\left(1 / \tau_{0}\right)(d \tau / d t)_{0} /\left[\left(\left(C_{2} / 2 C_{1}\right)-1\right\} N_{1}\right],
$$

where $\tau$ is turbidity (related to the transmission by $T=\exp (-\tau l)$ in a cuvette of length $l), t$ is time, and $C_{1}$ and $C_{2}$ are the extinction cross sections of a singlet and of a doublet, respectively. The optical factor $\left\{\left(C_{2} / 2 C_{1}\right)-1\right\}$ is calculated with the Rayleigh-Gans-Debye theory. The subscript zero refers to the limiting case of $t \rightarrow 0$.

The initial particle concentrations $N_{1}$ were determined from the dry weight of the latices. They were between $7.4 \times 10^{9}$ and $5.5 \times 10^{11} \mathrm{p}$ $\mathrm{cm}^{-3}$, where $\mathrm{p}$ is the number of particles. These values of $N_{1}$ were about $20 \%$ higher than those determined from turbidity with $\tau_{0}=C_{1} N_{1}$; only for one of the latices used $(2 \mathrm{a}=312 \mathrm{~nm})$ were both values equal. The disagreement between the dry weight values of $N_{1}$ and the turbidity values of $N_{1}$ cannot be explained by the presence of coagulated particles, since then the turbidity would overestimate $N_{1}$. Nor can it be explained by a small error in the particle radius; this would cause deviations of the same sign and of the same order of magnitude in $N_{1}$ as determined by turbidity and in $N_{1}$ as determined by dry weight. Probably optical imperfections of our system lead to underestimation of the turbidity. This is confirmed by theoretical (17) and experimental $(13,18)$ studies on this subject. The deviation was larger than expected from these studies. However, we assume that any systematic error in $\tau$ is present to the same extent in $d \tau / d t$, and thus cancels itself in $\left(1 / \tau_{0}\right)(d \tau / d t)_{0}$. This assumption is acceptable because Beer's law was valid in the range studied, which meant that any error in $\tau$, and thus in $d \tau / d t$ as well, would be a constant factor.

Measurements were performed at a wavelength (in air) of $546 \mathrm{~nm}$ with all latices, and also at some other wavelengths $(438,673$, and $752 \mathrm{~nm}$ ) with most latices. Changing the wavelength did not change the rate constant within the accuracy of the theory (16). We averaged the values of the rate constant obtained with various wavelengths.

Salts were used as received. Water was distilled twice; dissolved air was removed by boiling at low pressure before use. All experiments were performed at $20 \pm 1^{\circ} \mathrm{C}$.

\section{RESULTS}

The resulting values of $k_{11}$ for different particle sizes are given in Table $\mathrm{I}$. The reproduci- 
TABLE I

The Coagulation Rate Constant, Expressed as $k_{11}\left(\mathrm{p}^{-1} \mathrm{~cm}^{3} \mathrm{sec}^{-1}\right)$, and as a Percentage of THE VON SMOLUCHOWSKI VALUE

\begin{tabular}{|c|c|c|c|c|}
\hline \multirow[t]{2}{*}{ Experiments } & \multicolumn{2}{|c|}{$\begin{array}{c}\text { Dow } \\
\text { diameters } \\
(2 a)\end{array}$} & \multicolumn{2}{|c|}{$\begin{array}{l}\text { Dow } \\
\text { diameters } \\
\text { minus } 5 \%\end{array}$} \\
\hline & $k_{11} \times 10^{12}$ & $\%$ & $k_{11} \times 10^{12}$ & $\%$ \\
\hline $2 a=91 \mathrm{~nm}$ & 6.4 & 60 & 5.2 & 49 \\
\hline $2 a=109 \mathrm{~nm}$ & 5.9 & 55 & 4.7 & 44 \\
\hline $2 a=176 \mathrm{~nm}$ & 6.8 & 63 & 5.6 & 52 \\
\hline $2 a=234 \mathrm{~nm}$ & 5.3 & 50 & 4.5 & 42 \\
\hline $2 a=312 \mathrm{~nm}$ & 3.9 & 37 & 3.3 & 32 \\
\hline $2 a=357 \mathrm{~nm}$ & 5.5 & 51 & 4.6 & 43 \\
\hline Average $^{a}$ & 6.0 & 56 & 4.9 & 46 \\
\hline Theory & & & & \\
\hline$A=7.0 \times 10^{-21} \mathrm{~J}(12)$ & 6.0 & 56 & & \\
\hline$A=1.5 \times 10^{-21} \mathrm{~J}(12)$ & & & 4.9 & 46 \\
\hline von Smoluchowski & 10.8 & 100 & 10.8 & 100 \\
\hline
\end{tabular}

${ }^{a}$ Results of the $312-\mathrm{nm}$ latex have not been included in the average.

bility for the results was about $15 \%$. Though there is no complete agreement for different particle sizes, there is no clear dependence between $k_{11}$ and $a$. This is in agreement with the coagulation theory, which predicts that $k_{11}$ is independent of $a$, even when hydrodynamic interaction is considered $(11,12)$.

The particle radius (a) was given by Dow from their electron-microscopic measurements. The accuracy of these values is much disputed in literature (19-24). Many workers have reported somewhat lower values typically by about $5 \%$, obtained with different methods. Lowering the radius by $1 \%$ gives a mass per particle that is $3 \%$ lower; thus it raises $N_{1}$ by $3 \%$ for a given dry weight. As it also raises the optical factor $\left\{\left(C_{2} / 2 C_{1}\right)-1\right\}$, the resulting $k_{11}$ is over $3 \%$ lower according to Eq. [2]. We recalculated $k_{11}$ for each latex assuming a $5 \%$ lower particle radius.

\section{DISCUSSION AND CONCLUSION}

The von Smoluchowski theory (9) considering only brownian motion predicts $k_{11}=10.77$ $\times 10^{-12} \mathrm{p}^{-1} \mathrm{~cm}^{3} \mathrm{sec}^{-1}$ for water at $20^{\circ} \mathrm{C}$. When van der Waals attraction and hydrodynamic interaction are taken into account $(11,12)$, $k_{11}$ is found to be $40-65 \%$ of the von Smoluchowski value, dependent on the Hamaker constant $(A)$. Double layer repulsion does not affect the rate of rapid coagulation, which follows from the observation that increasing the electrolyte concentration does not increase the rate of coagulation. In principle, the Hamaker constant can be calculated from the measured values of $k_{11}$, but $k_{11}$ is rather insensitive to variation of $A$; doubling $A$ raises $k_{11}$ by about $10 \%$.

There is no complete agreement between theoretical calculations of the Hamaker constant by various authors using the Lifshitz theory, partly because of incomplete spectroscopic data. Krupp, Schnabel, and Walter (25) give $A=3.5 \times 10^{-21} \mathrm{~J}$ for the polystyrenewater system. Gingell and Parsegian (26) find $A=9.0 \times 10^{-21} \mathrm{~J}$ at interparticle distances shorter than $10 \mathrm{~nm}$, while at longer distances retardation lowers $A$ gradually to the limiting value of $A=3.2 \times 10^{-21} \mathrm{~J}$ at very large separation. For our particles we can use the nonretarded value, since attraction energy is very small compared to the energy of brownian motion at distances where retardation is important. Smith, Mitchell, and Ninham (27) find $A=11.6 \times 10^{-21} \mathrm{~J}$ for short separations. Referring to unpublished data of Parsegian and Ninham, they indicate that conduction processes in salt water may attenuate the microwave contribution by a factor $\exp (-\kappa d)$, where $d$ is the distance between particle surfaces, and $1 / \kappa$ is the Debye length. Assuming complete damping, as would be justified for our system where $1 / \kappa=0.5 \mathrm{~nm}$, they find $A=8.7 \times 10^{-21} \mathrm{~J}$. Evans and Napper (28) find $A=$ about $5 \times 10^{21} \mathrm{~J}$ for short distances; without the microwave contribution they find $A=3.5 \times 10^{-21} \mathrm{~J}$. They use a lower ionization potential for polystyrene in their calculations than some of the above authors $(26,27)$, who use the ionization potential of gaseous styrene. Using the latter (thus mistaken) value, Evans and Napper would find $A=\sim 7 \times 10^{-21} \mathrm{~J}$. 
We find $k_{11}=6.0 \times 10^{-12} \mathrm{p}^{-1} \mathrm{~cm}^{3} \mathrm{sec}^{-1}$ as an average over all experiments (the results of the latex with $2 a=312 \mathrm{~nm}$, which are much lower than the other ones, are left out of consideration); this value corresponds to $A=7.0$ $\times 10^{-21} \mathrm{~J}(12)$, in good agreement with the predictions made by the Lifshitz theory. If the sizes of all particles used were $5 \%$ lower, we would find $k_{11}=4.9 \times 10^{-12} \mathrm{p}^{-1} \mathrm{~cm}^{3} \mathrm{sec}^{-1}$, which corresponds to $A=1.5 \times 10^{-21} \mathrm{~J}$, somewhat lower than the theoretical values.

The standard deviation (about $5 \%$ ) of our average $k_{11}$ is smaller than the possible systematic error (about 18\%) in the case of a $5 \%$ overestimation of all particle radii.

Apart from giving a fair estimate of $A$, our results confirm strongly the theory of hydrodynamic interaction. Old determinations of $k_{11}$ for gold and selenium hydrosols (1a, 2) give values that are much higher, even higher than the von Smoluchowski value. Our turbidimetric results $(46-56 \%$ of the von Smoluchowski value) are in good agreement with recent lightscattering work of Lips, Smart, and Willis on polystyrene latex in water (6), who have found a rate constant of $67-69 \%$ of the von Smoluchowski value, and with recent low-angle light-scattering data of Lips and Willis (7), who have found $48-62 \%$ of the von Smoluchowski value.

In conclusion, the stopped-flow spectrophotometer makes it possible to observe latex coagulation in its first stage which is usually dealt with in theory. We find a rate constant that is in good agreement with calculations based on brownian motion, on hydrodynamic interaction, and on van der Waals attraction with a reasonable Hamaker constant.

\section{ACKNOWLEDGMENTS}

We thank Professor J. Th. G. Overbeek and G. J. Roebersen for helpful discussions.

\section{REFERENCES}

1. Overbeek, J. Th. G., in "Colloid Science" (H. R. Kruyt, Ed.), Vol. I, pp. 292-295 and references therein (a), p. 283 (b), p. 282 (c), Elsevier, Amsterdam, 1952.
2. Watillon, A., Romerowski, M., And GronderBeEck, F. van, Bull. Soc. Chim. Belges 68, 450 (1959).

3. Suitr, A. L., Spec. Discuss. Faraday Soc. 1, 032 (1970).

4. HiguchI, W. Y., Okada, R., Stelter, G. A., and Lemberger, A. P., J. Pham. Sci. 52, 49 (1963).

5. Matrhews, B. A., and Rhodes, C. T., J. Colloid Interface Sci. 32, 332 (1970).

6. Lips, A., Smart, C., and Willis, E., Trans. Farday Soc. 67, 2979 (1971).

7. Lips, A., ANd Willis, E., J. Chem. Soc. Faraday 69, 1226 (1973).

8. ReERinK, H., Thesis, p. 43. Utrecht, 1952.

9. SMoluchowski, M. von, Physik. Z. 17, 557 (1916); 17, 585 (1916); Z. Phys. Chem. 92, 129 (1917).

10. Fuchs, N., Z. Phys, 89, 736 (1934).

11. Setelman, L. A., J. Colloid Interface Sci. 33, 562 (1970).

12. Honig, E. P., Roebersen, G. J., ANd Wiersema, P. H., J. Colloid Interface Sci. 36, 97 (1971).

13. Heller, W., and Tabibian, R. M., J. Colloid Sci. 12, 25 (1957).

14. Vanderhoff, J. W., Hul, H. J. van den, Tausk, R. J. M., And Overbeek, J. Th. G., in "Clean Surfaces: Their Preparation and Characterization for Interfacial Studies" (G. Goldfinger, Ed.), p. 15. Marcel Dekiker, New York, 1970.

15. Roebersen, G. J., and Wiersema, P. H., $J$. Colloid Interface Sci., in press.

16. Lichtendelt, J. W. Th., Ras, H. J. M. C., and Wiersema, P. H., J. Colloid Interface Sci, 46, 522 (1974).

17. Latimer, P., J. Opt. Soc. Amer. 62, 208 (1972).

18. Walstra, P., Brit. J. Appl. Phys. 16, 1187 (1965).

19. DežžLic, G., and Kratohvid, J. P., J. Colloid Sci. 16, 561 (1961).

20. Philitps, D. T., Wyatt, P. J., and Berkman, R. M., J. Colloid Interface Sci. 34, 159 (1970).

21. Hut, H. J. van den, and Vanderhoff, J. W., in "Polymer Colloids" (R. M. Fitch, Ed.), p. 1. Plenum, New York, 1971.

22. Davidson, J. A., and Coltins, E. A., J. Colloid Interface Sci. 40, 437 (1972).

23. Lee, S. P., Tscharnuter, W., and Chu, B., $J$. Polym. Sci. A2 10, 2453 (1972).

24. COOKE, D. D., AND KeRKER, M., J. Colloid Interface Sci. 42, 150 (1973).

25. Krupp, H., Schnabei, W., and Walter, G., $J$. Colloid Interface Sci. 39, 421 (1972).

26. Gingeli, D., and Parsegian, V. A., J. Colloid Interface Sci. 44, 456 (1973).

27. Simth, E. R., Mrtcheli, D. J., and Ninham, B. W., J. Colloid Interface Sci. 45, 55 (1973).

28. Evans, R., and NAPPER, D. H., J. Colloid Interface Sci. 45, 138 (1973). 